\title{
ON A MODEL FOR INTERFERENCE BETWEEN SEARCHING INSECT PARASITES
}

\author{
P. K. POLLETT ${ }^{1}$
}

(Received January 1990)

\begin{abstract}
The purpose of this paper is to study a stochastic model which assesses the effect of mutual interference on the searching efficiency in populations of insect parasites. By looking carefully at the assumptions which govern the model, I shall explain why the searching efficiency is of the same order as the total number, $N$, in the population, a conclusion which is consistent with the predictions of population biologists; previous studies have reached the conclusion that the efficiency is of order $\sqrt{N}$. The major results of the paper establish normal approximations for the distribution of the numbers of active parasites. These are valid at all stages of the process, in particular the non-equilibrium phase, where explicit analytic formulae for the state-probabilities are unavailable.
\end{abstract}

\section{Introduction}

Recently, Diamond [5] described a one and two-species model for studying the effect of mutual interference between insect parasites on their searching efficiency. Diamond established a connection between stochastic models and their deterministic analogues. He showed that, in the one-species case, the equilibrium mean proportion of parasites actively searching for a host converges to a unique stable equilibrium point as the total number of parasites becomes large. For the two-species model, the corresponding result was established subject to a balance condition; under a slightly weaker condition Diamond showed that the deterministic model has a unique equilibrium in the positive quadrant, and that this is asymptotically stable. Since the one-species stochastic model is a birth-and-death process, the equilibrium

\footnotetext{
'Department of Mathematics, University of Queensland, Queensland 4072.

(C) Copyright Australian Mathematical Society 1990, Serial-fee code 0334-2700/90
} 
distribution is easy to write down. In the two-species case this appears not to be possible in general. However, Diamond was able to provide an explicit expression for the equilibrium distribution under the assumption that the process is "reversible". In doing so he established a role for hypergeometric functions similar to that revealed by McQuarrie [16] in his work on the stochastic approach to studying the kinetics of reversible chemical reactions. Unfortunately, the existence of explicit expressions for the equilibrium probabilities is of little comfort to the practitioner because, when the number of parasites is large, the computational problems are forbidding. This apparent anomaly that, in some cases, exact analytical expressions have only limited practical value, has been discussed by a number of authors (see, for example, Dunstan and Reynolds [6], [7] for a discussion in connection with chemical kinetic models) and often simulation is the preferred option (see, for example, Gillespie [8]). Another option, and this is the approach which I shall adopt in the present paper, is to use normal (Gaussian) approximations. These are extraordinarily simple to use, and they are extremely accurate if the number of parasites is reasonably large. They bear a relationship with Diamond's convergence results which is analogous to the relationship between the central limit theorem and the weak law of large numbers. Indeed it is useful for one to think of the relationship in these terms.

In this paper I shall establish a Gaussian approximation for the equilibrium state-probabilities which is also valid in the absence of Diamond's reversibility condition, that is, when exact analytical expressions are unavailable. Further, I shall provide a time-dependent approximation which is valid in the non-equilibrium phase. This will be achieved using the remarkable results of Kurtz [12], [13] and Barbour [2], [3], which allow one to show that the underlying Markov process can be approximated (in law) by an Ornstein-Uhlenbeck (OU) process. One immediate consequence is that Diamond's convergence results can be extended, without difficulty, to deal with the non-equilibrium phase. I note here that Diamond states that Kurtz's conditions for approximation by a deterministic model are not satisfied, for the transition rates are not "density dependent". However, a more careful examination of the modelling procedure reveals that Kurtz's work can be used in the present context, provided one interprets density dependence in a wider sense than was introduced in [12]. The density dependence of the rates not only facilitates an OU approximation, but it allows one to deduce that the mean and the variance of the equilibrium distribution in the one-species case are of the same order as $N$, the total number of parasites, an observation which is consistent with that predicted by population biologists (see May [15]); Diamond obtains an order of $N^{\frac{1}{2}}$ (see also Quinn and MacGillivray [17]). I shall show that this 
asymptotic result extends to the two-species model. Further, I shall show that it is valid in the non-equilibrium phase.

In Section 2 I shall briefly describe, and comment on, Diamond's stochastic model in the one and two-species cases. In Section 3 I shall adapt some results of Kurtz [12], [13] to deal with a wide and interesting class of processes which I call asymptotically density dependent. I shall use the one-species model as a vehicle for illustrating the results. The Gaussian approximations for this model are similar to those which have been obtained by Hsu and Wang [10] in their study of the kinetics of bacterial adhesion. Hsu and Wang adopt a method, attributed to van Kampen [21], of approximating the solution to the forward equations by expanding terms using a Taylor series. This method, though widely used, is quite cumbersome in comparison with Kurtz's. However, it is possible that practitioners have found Kurtz's work impenetrable. I hope to convince the reader of the simplicity of his results. The two-species model will be studied in Section 4. I shall establish that there is a bivariate OU process which describes the fluctuations of the process about the trajectory determined by a deterministic model. However, the deterministic model I shall use differs slightly from Diamond's in that he models the numbers of active parasites, while I model their population density, or equivalently, the searching efficiencies. Of course, as Diamond points out, it is necessary for the numbers of parasites to be large in order that the numbers of "actives" be considered as a continuously differentiable function of time. Thus, under these conditions, there is little qualitative difference between the two models. However, the model I shall use always has a unique, asymptotically stable equilibrium point in the positive quadrant; no balance condition is required. Finally, in Section 5, I shall comment on the accuracy of the diffusion approximation and briefly describe some other fruitful approaches.

\section{Diamond's model}

I shall begin by describing the one-species stochastic model; further details can be found in [5] and the references contained therein. Suppose that there is a fixed total number, $N$, of parasites, a random number, $P$, of which are actively searching for a host. An active parasite is assumed to become passive (inactive) when it perceives the presence of, or actually encounters, another parasite, while, in the passive state, a parasite becomes active after a time that is exponentially distributed with mean $T$; this time is taken to be constant and equal to $T$ in the deterministic model. The stochastic model is obtained by imposing the usual Markovian structure on the process. Thus, in the time interval $(s, s+t)$, exactly one active parasite (if available) 
becomes passive with probability proportional to $P(P-1) t+o(t)$, since there are precisely $\left(\begin{array}{l}P \\ 2\end{array}\right)$ possible encounters of $P$ parasites, while exactly one passive parasite (if available) becomes active with a probability proportional to $(N-P) t / T+o(t)$, since there are $N-P$ passive parasites. The probability of more than one event in $(s, s+t)$ is $o(t)$. Under these assumptions, the (continuous-time) Markov chain, $(P(t), t \geq 0)$, describing the number of parasites which are active at time $t$, takes values in $S=\{0,1, \ldots, N\}$ and has transition rates $q(j, k), j, k \in S$, given by

$$
q(j, k)= \begin{cases}\lambda(N-j), & \text { if } k=j+1, \\ \frac{1}{2} \phi j(j-1), & \text { if } k=j-1, \\ 0, & \text { if }|k-j|>1\end{cases}
$$

where $\lambda=T^{-1}$ and $\phi$ is the rate of encounter of any given pair of active parasites. It is conventional to set $q(j, j)$ equal to $-q(j)$, where $q(j)$ is the rate out of state $j$, and which here is given by

$$
q(j)=\lambda(N-j)+\phi j(j-1) / 2 .
$$

Note that Diamond's parameter $b$ equals $\frac{1}{2} \phi$. Diamond assumes that $b$ is constant. However, it will usually depend on $N$ and the precise functional relationship is important in determining the limiting behaviour of the process as $N$ becomes large. I shall suppose that the encounter rate for given pairs of actives is inversely proportional to the area, $A$, of the parasite habitat. Then, in adopting the simplest kind of "homogeneous mixing" assumption, one which amounts to supposing that population density does not vary significantly with $A$, I shall assume that $N=O(A)$, or, equivalently, $\phi=O\left(N^{-1}\right)$. Thus $\mu$, given by $\mu=\phi N / 2(=b N)$, is a dimensionless quantity. The limiting procedure which I shall describe amounts to observing the population over a wider and wider area.

A major drawback of the model is that the role of the host in affecting the behaviour of active parasites is not taken into account. As Diamond points out, parasites usually respond to their host in a "patchy" environment of host dispersion. One possible refinement of the model, which would account for grouping behaviour, might be to incorporate aspects of Whittle's immigration process [23]; Whittle's clustering process (see for example [22]) might also be useful for studying the relative sizes of the patches. However, the purpose of the present model is to study only the functional response of active parasites to members of their own species and, in the two-dimensional version, to members of different species. 
It is clear that $(P(t), t \geq 0)$ is a birth-and-death process over a finite state-space consisting of an ephemeral state 0 and an irreducible class $C=$ $\{1,2, \ldots\}$. Thus, assuming that $\operatorname{Pr}(P(0)=0)=0$, the limiting distribution, $\pi=(\pi(j), j \in S)$, is given by $\pi(0)=0$ and

$$
\pi(j)=\pi(1) \prod_{i=2}^{j} \frac{q(i-1, i)}{q(i, i-1)}=\pi(1) \frac{\theta^{j-1}}{j !}\left(\begin{array}{c}
N-1 \\
j-1
\end{array}\right), \quad j \in C,
$$

where $\theta=\lambda N / \mu(=1 /(b T))$ and $\pi(1)$ is chosen so that $\pi$ is a proper probability distribution over $S$.

Diamond comments that the mean and the variance of this distribution are slightly unusual in that they are of order $N^{\frac{1}{2}}$, rather than of order $N$ as predicted by population biologists. However, this apparent anomaly can be resolved by observing that $b$ is of order $N^{-1}$. On reworking Diamond's argument one finds that the mean and variance are both of order $N$.

The two-species version of the model allows for the (interspecific) interaction between two kinds of parasites, as well as the (intraspecific) interaction between parasites of the same kind. Suppose that there are $N_{i}$ parasites of type $i, i=1,2, P_{i}$ of which are actively searching for a host. Using precisely the same reasoning as before, one arrives at a twodimensional Markovian model. The Markov chain, $(P(t), t \geq 0)$, where $P=\left(P_{1}, P_{2}\right)^{T}$, describing the numbers of active parasites at time $t$, takes values in $S=\left\{0,1, \ldots, N_{1}\right\} \times\left\{0,1, \ldots, N_{2}\right\}$ and the only nonzero transition rates are given by

$$
\begin{aligned}
& q\left(\left(j_{1}, j_{2}\right),\left(j_{1}+1, j_{2}\right)\right)=\lambda_{1}\left(N_{1}-j_{1}\right), \\
& q\left(\left(j_{1}, j_{2}\right),\left(j_{1}, j_{2}+1\right)\right)=\lambda_{2}\left(N_{2}-j_{2}\right), \\
& q\left(\left(j_{1}, j_{2}\right),\left(j_{1}-1, j_{2}\right)\right)=\phi_{11} j_{1}\left(j_{1}-1\right) / 2+\phi_{12} j_{1} j_{2}, \\
& q\left(\left(j_{1}, j_{2}\right),\left(j_{1}, j_{2}-1\right)\right)=\phi_{22} j_{2}\left(j_{2}-1\right) / 2+\phi_{21} j_{2} j_{1} .
\end{aligned}
$$

Here $\lambda_{i}=T_{l}^{-1}$, where $T_{i}$ is the expected time that a type- $i$ parasite spends in the passive state and $\phi_{i i}$ and $\phi_{i j}, i \neq j$, are, respectively, the intraspecific and the interspecific encounter rates for given pairs of parasites. Again, since the encounters involve only two parasites, the rates are inversely proportional to $A$. However, $A$ will be of the same order as $N=N_{1}+N_{2}$, and the limiting operation of sending $N$ to $\infty$ will give rise to different phenomena in accordance with the order relationship between $N_{1}$ and $N_{2}$. To be consistent with previous notation let $\mu_{i i}=\phi_{i i} N / 2$ and $\mu_{i j}=\phi_{i j} N$, be the corresponding dimensionless quantities; in Diamond's nomenclature

$$
b_{i i}=\phi_{i i} / 2=\mu_{i i} / N \text { and } b_{i j}=\phi_{i j}=\mu_{i j} / N \text {. }
$$

As for the one-species case, 0 is an ephemeral state and so it is necessary to assume that $\operatorname{Pr}(P(0)=0)=0$ in order to obtain a unique equilibrium 
distribution, $\pi$. Clearly $\pi(0,0)=0$. However, it appears that only in the reversible case can one write down an explicit expression for the equilibrium probabilities over the irreducible class $C=S \backslash\{(0,0)\}$. It is easy to check, using the Kolmogorov criterion (see, for example, Pollett [20] and the references contained therein), that the process is reversible if and only if $\mu_{11}=\mu_{12}\left(=\mu_{1}\right)$ and $\mu_{22}=\mu_{21}\left(=\mu_{2}\right)$, that is, as Diamond points out, if and only if each species cannot distinguish between individuals of its own and the other species. Then, by iterating the detail-balance equations, it is elementary to show that

$$
\begin{aligned}
& \pi\left(j_{1}, j_{2}\right)=\pi(1,1) \frac{\theta_{1}^{j_{1}-1}}{j_{1} !} \frac{\theta_{2}^{j_{2}-1}}{j_{2} !} \frac{\left(N_{1}-1\right) !\left(N_{2}-1\right) !}{\left(N_{1}-j_{1}\right) !\left(N_{2}-j_{2}\right) !\left(j_{1}+j_{2}+1\right) !}, \\
& \text { for } j_{1}, j_{2} \geq 1 \text {, }
\end{aligned}
$$

and

$$
\begin{array}{ll}
\pi\left(j_{1}, 0\right)=\pi\left(j_{1}, 1\right)\left(j_{1} / N_{2}\right) \theta_{2}^{-1}, & \text { for } j_{1} \geq 1, \\
\pi\left(0, j_{2}\right)=\pi\left(1, j_{2}\right)\left(j_{2} / N_{1}\right) \theta_{1}^{-1}, & \text { for } j_{2} \geq 1,
\end{array}
$$

where $\theta_{i}=\lambda_{i} N_{i} / \mu_{i}\left(=1 /\left(b_{i i} T_{t}\right)\right)$ and $\pi(1,1)$ is chosen so that $\pi$ is a proper probability distribution over $S$. This result is contained in Diamond's Proposition 5 . There, the normalising constant is shown to have a representation in terms of hypergeometric functions of two variables. This facilitates a remarkable arithmetical calculation which establishes that, as $N_{1}$ and $N_{2}$ tend to $\infty$, the equilibrium expected values of $P_{1}$ and $P_{2}$ are asymptotic to the unique equilibrium point of the deterministic model which lies in the positive quadrant. In Section 4 I shall extend this result to the non-equilibrium phase. Further, it will be clear that the result always holds good; reversibility is not needed as a premise. Unfortunately, the corresponding arithmetical calculations needed to determine the asymptotic behaviour of the variances and the covariance of $P_{1}$ and $P_{2}$ are forbidding. The diffusion approximation which I shall use allows one to study this behaviour.

\section{Density dependence and diffusion approximations}

In this section I shall introduce a notion of density dependence which is more general than that introduced by Kurtz [12]. Let $\left\{X^{(\nu)}(\cdot), \nu>0\right\}$ be a family of continuous-time Markov chains and suppose that $X^{(\nu)}(\cdot)$ takes values in $S^{(\nu)}$, a subset of $Z^{K}$, and has transition rates $q^{(\nu)}(j, k), j, k \in$ $S^{(\nu)}$. It is instructive to be mindful of the one-species model described above. 
Indeed, I shall use it to illustrate all of the results presented in this section. The one-species model gives rise to a family of Markov chains, $\left\{P^{(N)}(\cdot)\right\}$, indexed by $N \geq 1$, with $P^{(N)}(\cdot)$ taking values in $S^{(N)}=\{0,1,2 \ldots, N\}$ and having transition rates, $q^{(N)}(\cdot, \cdot)$, given by $(2.1)$.

Definition 3.1. Suppose that there exists an open set $E \subseteq R^{K}$ and a family, $\left\{f^{(\nu)}, \nu>0\right\}$, of continuous functions with $f^{(\nu)}: E \times Z^{K} \rightarrow R$ such that

$$
q^{(\nu)}(k, k+l)=\nu f^{(\nu)}(k / \nu, l), \quad l \neq 0,
$$

and $\sum_{l} l f^{(\nu)}(x, l)$ converges for all $x \in E$. Then the family of Markov chains is asymptotically density dependent if, in addition, there exists a function $F: E \rightarrow R^{K}$, such that $\left\{F^{(\nu)}\right\}$, given by $F^{(\nu)}(x)=\sum_{l} l f^{(\nu)}(x, l), x \in$ $E$, converges to $F$ on $E$.

For example, the family $\left\{P^{(N)}(\cdot)\right\}$ is asymptotically density dependent, because $f^{(N)}, N \geq 1$, defined on any finite open interval $E$ which contains $[0,1]$ and given by

$$
f^{(N)}(x, 1)=\lambda(1-x), \quad f^{(N)}(x,-1)=\mu x\left(x-\frac{1}{N}\right),
$$

satisfy (3.1) and $\left\{F^{(N)}\right\}$, given by

$$
F^{(N)}(x)=\lambda(1-x)-\mu x\left(x-\frac{1}{N}\right),
$$

converges to $F$, given by $F(x)=\lambda(1-x)-\mu x^{2}$.

Kurtz's definition of density dependence requires only that there exists a continuous function, $f: R^{K} \times Z^{K} \rightarrow R$, such that

$$
q^{(\nu)}(k, k+l)=\nu f(k / \nu, l), \quad l \neq 0,
$$

and (implicitly) $\sum_{l} l f(x, l)<\infty$ for all $x$. Thus, an asymptotically density dependent family of Markov chains is density dependent if $f^{(\nu)}$ (and hence $F^{(\nu)}$ ) does not depend on $\nu$. Observe that, although $\left\{P^{(N)}(\cdot)\right\}$ is asymptotically density dependent, it is not density dependent.

Roughly speaking, a family is density dependent if the transition rates of the corresponding "density process", $\frac{1}{\nu} X^{(\nu)}(\cdot)$, depend on the present state, $k$, only through the density $k / \nu$; an asymptotically density dependent family is one which exhibits this property in the limit as $\nu \rightarrow \infty$. Thus there is a natural way to associate with this process a density dependent deterministic process which, for large $\nu$, is "tracked" by the process. Indeed, a straightforward formal argument based on the forward equations,

$$
\frac{d}{d t} p^{(\nu)}(j ; t)=\sum_{i \in S^{(\nu)}} p^{(\nu)}(i ; t) q^{(\nu)}(i, j), \quad t>0,
$$


for $p^{(\nu)}(j ; t)=\operatorname{Pr}\left(X^{(\nu)}(t)=j\right), j \in S, t>0$, shows that, for large $\nu$,

$$
\frac{d}{d t} \mathbf{E}\left(\frac{1}{\nu} X^{(\nu)}(t)\right) \simeq \mathbf{E} F^{(\nu)}\left(\frac{1}{\nu} X^{(\nu)}(t)\right), \quad t>0 .
$$

Thus one might expect this deterministic process, call it $X(\cdot)$, to satisfy

$$
d X(t) / d t=F(X(t)), \quad t>0 .
$$

The following "law of large numbers" establishes that, under appropriate conditions, the density process does track a deterministic process. It is the analogue of Theorem 3.1 of Kurtz [12] for asymptotically density dependent families of processes, and, as the proof is similar, I shall omit the details.

THEOREM 3.1. Suppose that $F$ is Lipschitz continuous on $E$ and that for all $\nu>0$

$$
\begin{gathered}
\sup _{x \in E} \sum_{l}|l| f^{(\nu)}(x, l)<\infty, \\
\lim _{\delta \rightarrow \infty} \sup _{x \in E} \sum_{l:|l|>\delta}|l| f^{(\nu)}(x, l)=0
\end{gathered}
$$

and

$$
\lim _{\nu \rightarrow \infty} \sup _{x \in E}\left|F^{(\nu)}(x)-F(x)\right|=0
$$

Then, if

$$
\lim _{\nu \rightarrow \infty} \frac{1}{\nu} X^{(\nu)}(0)=x
$$

we have that

$$
\lim _{\nu \rightarrow \infty} \operatorname{Pr}\left(\sup _{s \leq t}\left|\frac{1}{\nu} X^{(\nu)}(s)-X(s, x)\right|>\varepsilon\right)=0, \quad 0 \leq s \leq t,
$$

for all $\varepsilon>0$, and for every trajectory $X(\cdot, x)$ satisfying

$$
\begin{gathered}
X(0, x)=x, \\
X(s, x) \in E, \quad 0 \leq s \leq t, \\
\frac{\partial}{\partial s} X(s, x)=F(X(s, x)) .
\end{gathered}
$$

REMARKS. (1) Conditions (3.3) and (3.4) will be trivially satisfied if $f^{(\nu)}$ is bounded (on $E$ ) and if there are only a finite number of transitions out of any state, $k$, for example if $S^{(\nu)}$ is finite; this is because $f^{(\nu)}(\cdot, l)$ will be equal to 0 for $|l|$ sufficiently large. Both of these conditions, together with the condition that $F$ be Lipschitz continuous, are satisfied in the example.

(2) Condition (3.6) stipulates that the density process should begin close to the initial value, $x$, of the deterministic trajectory. The conclusion (3.7) 
then states that at each $s$ in some appropriate time-interval, the largest deviation of the density process about its deterministic path converges in probability to 0 . Thus one can conclude that $\left\{\frac{1}{\nu} X^{(\nu)}(s)\right\}$ converges in probability to $X(s, x)$ and, if for each $s, X^{(\nu)}(s) / \nu$ is a.s. uniformly bounded, the Lebesgue dominated convergence theorem implies that

$$
\lim _{\nu \rightarrow \infty} \frac{1}{\nu} \mathbf{E} X^{(\nu)}(s)=X(s, x)
$$

is true on all time-intervals such that $X(\cdot, x)$ remains in $E$. In the example one can use the theorem to study the searching efficiency, $\bar{P}^{(N)}(\cdot)=$ $P^{(N)}(\cdot) / N$, that is, the relative abundance of active parasites. It is reasonable to suppose that $P^{(N)}(0)=N$ so that intially the searching efficiency is 1 , but for any initial value one can conclude that, for large $N$, the searching efficiency is asymptotic to the deterministic path. Since there is a unique equilibrium point in $(0,1)$, and this is asymptotically stable, we have that

$$
\lim _{N \rightarrow \infty} \operatorname{Pr}\left(\sup _{s \leq t}\left|\bar{P}^{(N)}(s)-P(s, x)\right|>\varepsilon\right)=0
$$

for all $\varepsilon>0$ and all $t>0$, where $P(\cdot, x)$ satisfies $P(0, x)=x$ and

$$
d P / d t=\lambda(1-P)-\mu P^{2}, \quad \text { where } \quad x=\lim _{N \rightarrow \infty} \bar{P}^{(N)}(0) .
$$

Thus, in particular, since for each $s, \bar{P}^{(N)}(s)$ is uniformly bounded, I have extended Diamond's result ([5], Proposition 1) to the non-equilibrium phase: $\lim _{N \rightarrow \infty} \mathbf{E} \bar{P}^{(N)}(s)=P(s, x)$, for all $s>0$.

(3) The parameter $\nu$, usually interpreted as the "size of the system", need not be discrete. For example, one could index $P(\cdot)$ by the area, $A$, of the parasite habitat, rather than by $N$. One would then be able to study the density, $P^{(A)}(\cdot) / A$, of "actives" as $A$ becomes large. The corresponding results can be obtained easily using a change of variable, $x \rightarrow x / \alpha$, where $\alpha$, the limiting population density, is given by $\alpha=\lim _{A \rightarrow \infty}(N(A) / A)$, so that all relevant quantities are scaled by $\alpha$.

The law of large numbers for an asymptotically density dependent process tells one that such a process can be approximated over any finite time-interval by a deterministic path defined on that interval. However, it does not tell one anything of the random fluctuations about this path. The following "central limit law" establishes that, for large $\nu$, these fluctuations follow a diffusion, provided that certain "second order" conditions are satisfied. Again I shall omit the proof. It follows from Theorem (3.1) and Theorem (3.5) of Kurtz [13]. 
THEOREM 3.2. Suppose that $\left\{F^{(\nu)}\right\}$ converges uniformly to $F$ and that $F$ is bounded and Lipschitz continuous on $E$. Suppose also that the family $\left\{G^{(\nu)}\right\}$, where $G^{(\nu)}(x)$ is a $K \times K$ matrix with elements

$$
g_{i j}^{(\nu)}(x)=\sum_{l} l_{i} l_{J} f^{(\nu)}(x, l), \quad x \in E,
$$

converges uniformly to $G$, where $G$ is bounded and uniformly continuous on $E$.

If, in addition,

$$
\begin{gathered}
\sup _{x \in E} \sum_{l}|l|^{2} f^{(\nu)}(x, l)<\infty, \\
\lim _{\delta \rightarrow \infty} \sup _{x \in E} \sum_{l:|l|>\delta}|l|^{2} f^{(\nu)}(x, l)=0,
\end{gathered}
$$

for all $\nu>0$, and

$$
\lim _{\nu \rightarrow \infty} \sup _{x \in E} \nu^{\frac{1}{2}}\left|F^{(\nu)}(x)-F(x)\right|=0,
$$

where now $F$ is assumed to have uniformly continuous first partial derivatives, then, provided

$$
\lim _{\nu \rightarrow \infty} \nu^{\frac{1}{2}}\left(\frac{1}{\nu} X^{(\nu)}(0)-x\right)=z,
$$

the family of processes $\left\{Z^{(\nu)}(\cdot)\right\}$, defined by

$$
Z^{(\nu)}(s)=\nu^{\frac{1}{2}}\left(\frac{1}{\nu} X^{(\nu)}(s)-X(s, x)\right), \quad 0 \leq s \leq t,
$$

converges weakly in $D[0, t]$ (the space of right-continuous, left-hand limits functions on $[0, t])$ to a diffusion, $Z(\cdot)$, with initial value $Z(0)=z$ and with characteristic function, $\psi=\psi(s, \theta)$, which satisfies

$$
\begin{aligned}
\frac{\partial \psi}{\partial s}(s, \theta)= & -\frac{1}{2} \sum_{j, k} \theta_{j} g_{j k}(X(s, x)) \theta_{k} \psi(s, \theta) \\
& +\sum_{j, k} \theta_{j} \frac{\partial F_{j}}{\partial x_{k}}(X(s, x)) \frac{\partial \psi}{\partial \theta_{k}}(s, \theta) .
\end{aligned}
$$

REMARK. (1) Conditions (3.8) and (3.9) will be trivially satisfied if $f^{(\nu)}$ is bounded and there are finitely many possible transitions out of each state.

(2) Condition (3.10) strengthens (3.5) to ensure that $\left\{F^{(\nu)}\right\}$ converges to $F$ at the correct rate. It is satisfied in the example because $\left|F^{(N)}(x)-F(x)\right|=$ $O\left(N^{-1}\right)$.

(3) Condition (3.11) provides the initial value of the diffusion. 
(4) Although (3.12) specifies the distribution of the diffusion, only in special cases can one obtain an explicit expression for its characteristic function. However, one can always determine the mean and variance of $Z(s)$ and thus, for large $\nu$, an approximate formula for the mean and variance of the density process, $X^{(\nu)}(\cdot) / \nu$. If one denotes by $\nabla F$ the matrix of the first partial derivatives of $F$, that is $\nabla F=\left[\partial F_{i} / \partial x_{j}\right]$, and puts $B_{s}=\nabla F(X(s, x))$, then $\mathbf{E} Z(s)=M_{s} z$, where $M_{s}$ is the unique solution to $d M_{s} / d s=B_{s} M_{s}$ with $M_{0}=I$, that is

$$
M_{s}=\exp \left(\int_{0}^{s} B_{u} d u\right)
$$

On the other hand, the convariance matrix, $\Sigma_{s}$, of $Z(s)$ is the unique solution to

$$
d \Sigma_{s} / d s=B_{s} \Sigma_{s}+\Sigma_{s} B_{s}^{\top}+G(X(s, x))
$$

with $\Sigma_{0}=0$, that is

$$
\Sigma_{s}=M_{s}\left(\int_{0}^{s} M_{u}^{-1} G(X(u, x))\left(M_{u}^{-1}\right)^{\top} d u\right) M_{s}^{\top} .
$$

In the example $G^{(N)}(x)=\lambda(1-x)+\mu x(x-(1 / N))$, and so it is clear that $\left\{G^{(N)}\right\}$ converges uniformly to $G$, given by $G(x)=\lambda(1-x)+\mu x^{2}$, and that $G$ is bounded and uniformly continuous on any suitable finite open interval, $E$. It is also clear that $\left\{F^{(N)}\right\}$ converges to $F$ uniformly on $E$, and that $F$ is bounded on $E$ with uniformly continuous derivative, $F^{\prime}$, given by $F^{\prime}(x)=-(\lambda+2 \mu x)$. Therefore, one can conclude that, provided $\lim _{N \rightarrow \infty} N^{\frac{1}{2}}\left(\bar{P}^{(N)}(0)-x\right)=z$, the family $\left\{Z^{(N)}(\cdot)\right\}$, given by

$$
Z^{(N)}(s)=N^{\frac{1}{2}}\left(\bar{P}^{(N)}(s)-P(s, x)\right),
$$

converges weakly to a diffusion, $Z(\cdot)$. The mean and the variance of $Z(s)$ can be calculated using expressions (3.13) and (3.14) and, although the integration is tricky, they can be evaluated explicitly. One finds that, to first order,

$$
M_{s}=e^{-\left(\lambda+2 \mu x_{0}\right) s}\left(1-2 \mu\left(x-x_{0}\right) s+O\left(s^{2}\right)\right)
$$

and

$$
\Sigma_{s}=\frac{G(x)}{2\left(\lambda+2 \mu x_{0}\right)}\left(1-e^{-2\left(\lambda+2 \mu x_{0}\right) s}\right)+O\left(s^{2}\right),
$$

where $x$ is the starting point of the deterministic trajectory and $x_{0}$ is the unique positive (asymptotically stable) equilibrium point. The deterministic trajectory is given by

$$
P(s, x)=\frac{x_{0}\left(x-x_{1}\right)-x_{1}\left(x-x_{0}\right) e^{-\alpha s}}{\left(x-x_{1}\right)-\left(x-x_{0}\right) e^{-\alpha s}}, \quad s \geq 0,
$$


where $\alpha=\mu\left(x_{0}-x_{1}\right)$ and $x_{1}$ is the negative equilibrium point. An approximation for the mean and variance of the searching efficiency can thus be obtained: for large $N$,

$$
\mathbf{E} \bar{P}^{(N)}(s) \simeq P(s, x)+M_{s}\left(\bar{P}^{(N)}(0)-x\right) \quad \text { and } \quad \operatorname{Var} \bar{P}^{(N)}(s) \simeq N^{-1} \Sigma_{s} .
$$

Observe that the mean and variance of the number of active parasites at time $s$ are both of order $N$. Thus, the observation made in Section 2 concerning the mean and variance of the equilibrium distribution is also valid in the non-equilibrium phase.

(4) I have mentioned that, in general, (3.12) does not readily yield the distribution of $Z(s)$. However, it does in the important special case where $x$ is chosen as an equilibrium point, $x_{0}$, of (3.2). If (3.11) is satisfied, then $\left\{Z^{(\nu)}(\cdot)\right\}$ converges to an OU process, $Z(\cdot)$, with local drift matrix $B=$ $\nabla F\left(x_{0}\right)$ and local covariance matrix $G\left(x_{0}\right)$. In particular, it is a Gaussian diffusion, that is $Z(s)$ is normally distributed. From (3.13) and (3.14) it can be seen that the mean of $Z(s)$ is given by $\mathbf{E} Z(s)=e^{B s} z$ and the covariance matrix by

$$
\Sigma_{s}=e^{B s}\left(\int_{0}^{s} e^{-B u} G\left(x_{0}\right) e^{-B^{\top} u} d u\right) e^{B^{\top} s} .
$$

In the one-species case, $K=1$, all quantities are scalars or scalar functions, and (3.17) reduces to $\operatorname{Var} Z(s)=\left(G\left(x_{0}\right) /(2 B)\right)\left(e^{2 B s}-1\right)$. It should be emphasised that $x_{0}$ need not be asymptotically stable. Indeed, the OU approximation is often very accurate in describing the fluctuations about centres and unstable equilibria (see Barbour [3]). This is particularly important when the underlying stochastic model exhibits apparent equilibrium behaviour near an unstable deterministic equilibrium; this phenomenon is known as quasi-stationarity (see, for example, Parsons and Pollett [18] and Pollett [19]).

If, in the example, one takes $x_{0}$ to be the (asymptotically stable) positive equilibrium point, so that $B=F^{\prime}\left(x_{0}\right)=-\left(\lambda+2 \mu x_{0}\right)<0$ and $G\left(x_{0}\right)=$ $\lambda\left(1-x_{0}\right)+\mu x_{0}^{2}=2 \mu x_{0}^{2}$, then it is easy to see that, for large $N$, the searching efficiency at time $s$ is approximately normally distributed with mean and variance given by

$$
\mathbf{E} \bar{P}^{(N)}(s) \simeq x_{0}+e^{-\left(\lambda+2 \mu x_{0}\right) s}\left(\bar{P}^{(N)}(0)-x_{0}\right)
$$

and

$$
\operatorname{Var} \bar{P}^{(N)}(s) \cong N^{-1} \frac{\mu x_{0}^{2}}{\lambda+2 \mu x_{0}}\left(1-e^{-2\left(\lambda+2 \mu x_{0}\right) s}\right) .
$$

Compare these expressions with the approximations obtained from (3.15) and (3.16). It might at first appear that they are the same to first order in 
$s$. However, although the expected value agrees to first order, this is not the case for the variance, because observe carefully that it is $G(x)$, rather than $G\left(x_{0}\right)$ which appears in (3.16).

\section{A Gaussian approximation for the two-species model}

In the two-species case, there are a number of ways that one can index the Markov chain $(P(t), t \geq 0)$. If $N$, the total number of parasites, is chosen as the index, an asymptotically density dependent family, $\left\{P^{(N)}(\cdot), N \geq 1\right\}$, is obtained, for, in accordance with Definition 3.1, one can define a sequence, $\left\{f^{(N)}\right\}$, of continuous functions, with $f^{(N)}: R^{2} \times Z^{2} \rightarrow R$, which satisfy (3.1). These are given by

$$
f^{(N)}(x, l)= \begin{cases}\lambda_{1}\left(\alpha_{1}-x_{1}\right) & \text { if } l=(1,0), \\ \lambda_{2}\left(\alpha_{2}-x_{2}\right) & \text { if } l=(0,1), \\ \mu_{11} x_{1}\left(x_{1}-1 / N\right)+\mu_{12} x_{1} x_{2} & \text { if } l=(-1,0), \\ \mu_{22} x_{2}\left(x_{2}-1 / N\right)+\mu_{21} x_{2} x_{1} & \text { if } l=(0,-1),\end{cases}
$$

where $\alpha_{1}$ and $\alpha_{2}$, the limiting proportions of the two species, are given by

$$
\alpha_{i}=\lim _{N \rightarrow \infty}\left(N_{i} / N\right), \quad i=1,2 ;
$$

one can allow $N_{1}$ and $N_{2}$ to be of different order as $N \rightarrow \infty$, in which case one of the $\alpha$ 's will be equal to 0 and the other equal to 1. The set $E$ can be any finite open set which contains the rectangle $H=\left[0, \alpha_{1}\right] \times\left[0, \alpha_{2}\right]$. The sequence of functions $\left\{F^{(N)}\right\}$ is given by

$$
F^{(N)}(x)=\left(\begin{array}{l}
\lambda_{1}\left(\alpha_{1}-x_{1}\right)-\mu_{11} x_{1}\left(x_{1}-1 / N\right)-\mu_{12} x_{1} x_{2} \\
\lambda_{2}\left(\alpha_{2}-x_{2}\right)-\mu_{22} x_{2}\left(x_{2}-1 / N\right)-\mu_{21} x_{2} x_{1}
\end{array}\right)
$$

and the function, $F$, to which it converges uniformly on $E$ is given by

$$
F(x)=\left(\begin{array}{l}
\lambda_{1}\left(\alpha_{1}-x_{1}\right)-\mu_{11} x_{1}^{2}-\mu_{12} x_{1} x_{2} \\
\lambda_{2}\left(\alpha_{2}-x_{2}\right)-\mu_{22} x_{2}^{2}-\mu_{21} x_{2} x_{1}
\end{array}\right) .
$$

The appropriate deterministic model that one should consider is as follows:

$$
d P(t) / d t=F(P(t)), \quad t>0 .
$$

Before proceeding to study the asymptotic behaviour of the stochastic model, I shall explain why the deterministic model has a unique, asymptotically stable equilibrium point in the positive quadrant.

One can easily adapt Diamond's argument, based on the isoclines determined by $d P_{1} / d t=0$ and $d P_{2} / d t=0$, to show that there is at least one equilibrium point in the positive quadrant, and that this lies in the rectangle $H$; one requires a modicum of artistic skill (in the case provided by 
MacGillivray) in order to be convinced that, a priori, there can be two or three such equilibria. I shall establish that the equilibrium point is, in fact, unique by proving that all positive equilibria must be asymptotically stable. Once this is done, I can choose the set $E$ so that any trajectory, $P(\cdot, x)$, which starts in $E$ will remain there.

Let $x_{0}$ be an arbitrary equilibrium point in the positive quadrant and rewrite (4.1) as $d P / d t=B\left(P-x_{0}\right)-h\left(P-x_{0}\right)$, where $B=\nabla F\left(x_{0}\right)$ and $h$ is given by

$$
h(x)=\left(\begin{array}{l}
\mu_{11} x_{1}^{2}+\mu_{12} x_{1} x_{2} \\
\mu_{22} x_{2}^{2}+\mu_{21} x_{2} x_{1}
\end{array}\right) .
$$

Then, it is easy to verify that $B$ is negative definite and that $\|h(x)\|=$ $o(\|x\|)$, as $\|x\| \rightarrow 0$. So, using classical stability theory (see, for example, Theorem 9.6 of Jordan and Smith [11]), one can deduce that $x_{0}$ is asymptotically stable.

The conditions of Theorem 3.1 are easily verified: (3.3) and (3.4) are trivially satisfied because the $f^{(N)}$ 's are bounded on $E$ and there are finitely many transitions out of each state, while (3.5) is satisfied because

$$
F^{(N)}(x)-F(x)=N^{-1}\left(\begin{array}{l}
\mu_{11} x_{1} \\
\mu_{22} x_{2}
\end{array}\right) .
$$

It follows that if $\lim _{N \rightarrow \infty} P^{(N)}(0) / N=x$, then, for all $t>0$,

$$
\lim _{N \rightarrow \infty} \operatorname{Pr}\left(\sup _{s \leq t}\left|P^{(N)}(s) / N-P(s, x)\right|>\varepsilon\right)=0, \quad 0 \leq s \leq t,
$$

for each $\varepsilon>0$. In particular, since for each $s, P^{(N)}(s) / N$ is uniformly bounded, the ensuing convergence in probability of $\left\{P^{(N)}(s) / N\right\}$ to $P(s, x)$ implies that

$$
\lim _{N \rightarrow \infty} \mathbf{E}\left(P^{(N)}(s) / N\right)=P(s, x),
$$

for all $s>0$. The expected value of the searching efficiency,

$$
\bar{P}_{i}^{(N)}(s)=P_{i}^{(N)}(s) / N,
$$

of type- $i$ parasites is therefore asymptotic to $\alpha_{i}^{-1} P_{i}(s, x)$.

I shall now consider the fluctuations of the stochastic model about the deterministic path, $P(\cdot, x)$. The conditions of Theorem 3.2 are all satisfied with no extra assumptions. Again (3.8) and (3.9) are trivially satisfied for reasons previously mentioned, and (3.10) holds because $\left|F^{(N)}(x)-F(x)\right|=$ $O\left(N^{-1}\right) . F$ is clearly bounded and Lipschitz continuous on $E$ and $\nabla F$, given by

$$
\nabla F(x)=\left(\begin{array}{cc}
-\lambda_{1}-2 \mu_{11} x_{1}-\mu_{12} x_{2} & -\mu_{12} x_{1} \\
-\mu_{21} x_{2} & -\lambda_{2}-2 \mu_{22} x_{2}-\mu_{21} x_{1}
\end{array}\right),
$$


is uniformly continuous on $E$. The sequence $\left\{G^{(N)}\right\}$, given by $G^{(N)}(x)=\left(\begin{array}{cc}\lambda_{1}\left(\alpha_{1}-x_{1}\right)+\mu_{11} x_{1}\left(x_{1}-\frac{1}{N}\right)+\mu_{12} x_{1} x_{2} & 0 \\ 0 & \lambda_{2}\left(\alpha_{2}-x_{2}\right)+\mu_{22} x_{2}\left(x_{2}-\frac{1}{N}\right)+\mu_{21} x_{2} x_{1}\end{array}\right)$, converges uniformly to $G$, given by

$$
G(x)=\left(\begin{array}{cc}
\lambda_{1}\left(\alpha_{1}-x_{1}\right)+\mu_{11} x_{1}^{2}+\mu_{12} x_{1} x_{2} & 0 \\
0 & \lambda_{2}\left(\alpha_{2}-x_{2}\right)+\mu_{22} x_{2}^{2}+\mu_{21} x_{2} x_{1}
\end{array}\right) .
$$

Clearly $G$ is also bounded and uniformly continuous on $E$. One can therefore assert that, provided $\lim _{N \rightarrow \infty} N^{\frac{1}{2}}\left(P^{(N)}(0) / N-x\right)=z$, the family of processes $\left\{Z^{(N)}(\cdot)\right\}$ given by

$$
Z^{(N)}(s)=N^{\frac{1}{2}}\left(P^{(N)}(s) / N-P(s, x)\right), \quad 0 \leq s \leq t,
$$

converges weakly to a diffusion, $Z(\cdot)$. Although one cannot hope to obtain explicit expressions for the mean and the covariance matrix of $Z(s)$, nor indeed for the deterministic trajectory, this result does lead to the conclusion that the mean and the variance of $P_{i}(s)$, the number of type- $i$ parasites, as well as the covariance of $P_{1}(s)$ and $P_{2}(s)$ are all of order $N$.

The OU approximation for the two-species model is obtained on choosing $x$ to be the equilibrium point $x_{0}$. The sequence $\left\{Z^{(N)}(\cdot)\right\}$ then converges to an OU process, $Z(\cdot)$, with local drift matrix $B=\nabla F\left(x_{0}\right)$ and local covariance matrix $G=G\left(x_{0}\right)$. The integration in (3.17) can, of course, be performed without difficulty since all matrices are $2 \times 2$. However, it is customary (see Barbour [3]) and, in practice, certainly more convenient, to change coordinates by putting

$$
W^{(N)}(s)=A Z^{(N)}(s),
$$

where the rows of $A$ are the left-eigenvectors of $B$. The resulting sequence, $\left\{W^{(N)}(\cdot)\right\}$, converges weakly to an OU process, $W(\cdot)$, whose individual components are themselves OU processes. Its local drift matrix is $D=\operatorname{diag}\left(\eta_{1}, \eta_{2}\right)$, where $\eta_{1}$ and $\eta_{2}$ are the (strictly negative and distinct) eigenvalues of $B$, and its local covariance matrix is $S=A G A^{\top}$. In particular, $W(s)$ has a bivariate normal distribution with

$$
\mathbf{E} W_{i}(s)=w_{i} e^{\eta_{i} s}, \quad \operatorname{Var} W_{i}(s)=S_{i i}\left(e^{2 \eta_{i} s}-1\right) /\left(2 \eta_{i}\right)
$$

and

$$
\operatorname{Cov}\left(W_{1}(s), W_{2}(s)\right)=S_{12}\left(e^{\left(\eta_{1}+\eta_{2}\right) s}-1\right) /\left(\eta_{1}+\eta_{2}\right),
$$

where $w=A z$. The searching efficiencies, $\bar{P}_{1}(s)$ and $\bar{P}_{2}(s)$, have an 
approximate bivariate normal distribution whose parameters are easily recovered by inverting (4.2).

\section{Concluding remarks}

On the accuracy of the diffusion approximation. There are a number of results that allow one to assess the accuracy of the diffusion approximation, in particular, ones which show how closely, for how long and over what ranges the "density process" is faithfully approximated. For example, Barbour [4] reports (summarising results of Kurtz [14] and Alm [1], respectively) that there is a version of the diffusion, $Z(\cdot)$, defined on the same probability space as $\left\{X^{(\nu)}(\cdot)\right\}$ such that, for all $t>0$,

$$
\sup _{s \leq t}\left|Z^{(\nu)}(s)-Z(s)\right|=O\left(\nu^{-\frac{1}{2}} \log \nu\right)
$$

and that the distribution of $Z^{(\nu)}(s)$ converges to that of $Z(s)$ at the "natural" rate of $\nu^{-\frac{1}{2}}$. The conditions needed for these results to hold are satisfied in the present context.

With reference to the OU approximation, it is possible to obtain explicit results concerning the order of the error arising from approximating the distribution of $Z^{(\nu)}(s)$ by a normal distribution (see Barbour [3]). For example, in the one-dimensional case, where the OU process has mean $m(s)=e^{B s} z$ and variance $v(s)=G\left(x_{0}\right)\left(e^{2 B s}-1\right) /(2 B)$, it can be shown that if $x_{0}$ is asymptotically stable,

$$
\operatorname{Pr}\left(Z^{(\nu)}(s) \geq x\right)=\left(1-\Phi\left(\frac{x-m(s)}{\sqrt{v}(s)}\right)\right)\left(1+O\left(M^{4} \nu^{-\frac{1}{2}} \log \nu\right)\right),
$$

where $\Phi$ is the standard normal distribution function, provided $M$ is of order between $(\log \nu)^{\frac{1}{2}}$ and $\nu^{\frac{1}{8}}$. This result holds uniformly over $x$ in $[0, M]$ and over time-intervals of the form $\left[s_{0}, \exp \left(\delta M^{2}\right)\right]$, for any $s_{0}, \delta>$ 0 . Thus, the questions of "how closely" and "over what ranges" are both answered here.

Barbour also obtains results which show that, when $x_{0}$ is asymptotically stable, the time till first exit of the density process, $X^{(\nu)}(\cdot) / \nu$, from an interval of the form $\left\{x:\left|x-x_{0}\right|<\nu^{-\frac{1}{2}} c_{\nu}\right\}$, where $\left\{c_{\nu}\right\}$ converges to $\infty$, is approximately exponentially distributed, conditional on the process leaving at, say, the right endpoint. This result answers the question of "how long", because it establishes that, provided $c_{\nu}=o\left(\nu^{\frac{1}{8}}\right)$, the mean time till exit is asymptotically $\frac{1}{2 B c_{\nu}} \sqrt{\frac{\pi G\left(x_{0}\right)}{-B}} e^{-B c_{\nu}^{2} / G\left(x_{0}\right)}$. Thus, in the one-species model, the 
time taken for $\bar{P}^{(N)}(\cdot)$ to first leave the interval $\left\{x:\left|x-x_{0}\right|<N^{-\frac{1}{2}} c_{N}\right\}$ is of order $c_{N}^{-1} \exp \left(\kappa c_{N}^{2}\right)$, where $\kappa=\left(\lambda+2 \mu x_{0}\right) /\left(2 \mu x_{0}^{2}\right)$, whenever $c_{N}=\left(N^{\frac{1}{8}}\right)$. Hence, it is asymptotically larger than any power of $N$ if, for example, $c_{N}=O\left(N^{\frac{1}{8}} / \log N\right)$. Barbour also provides analogous results which are appropriate for dealing with the two-species model. These indicate for how long the OU process $W(\cdot)$ provides an adequate distributional approximation for $W^{(N)}(\cdot)$.

Other approximations. It is possible to obtain normal and other distributional approximations when the density dependence condition is relaxed. Recall that, in the model, this condition arises naturally out of the assumption that the encounter rate is inversely proportional to $N$, and it allows one to deduce asymptotic results which are consistent with the predictions of population biologists. However, if empirical evidence (and I am aware of none) were to suggest that the encounter rate does not vary with $N$, as in [5], then, under this assumption, the equilibrium distribution (2.2) would be approximately normal with mean $(N \theta)^{\frac{1}{2}}+O(1)$ and variance $(N \theta / 4)^{\frac{1}{2}}+O\left(N^{-\frac{1}{2}}\right)$, where $\theta=2 \lambda / \phi(=1 /(b T))$ (see Quinn and MacGillivray [17]). This result can also be obtained by adapting the proof of Theorem (2.5) (i) of Hall [9]. In fact, one can mimic all of the limiting operations performed by Hall on the equilibrium distribution of the numbers of molecules in the simple chemical reaction scheme $A+B \rightleftharpoons C$. For example, if $N \rightarrow \infty$ and $\theta \rightarrow 0$ in such a way that $\theta N=m$, one obtains a Bessel distribution (with index 1 and with parameter $m$ ) as an approximation to the equilibrium distribution of the number of active parasites. If, instead, $\theta \rightarrow \infty$ and $N \rightarrow \infty$ in such a way that $N / \theta=m$, then the equilibrium distribution of the number of passive parasites is approximately Poisson with mean $\mathrm{m}^{2}$. These approximations are appropriate for describing the equilibrium behaviour of large populations where the encounter rate is large, respectively, small in comparison with the rate at which parasites become active.

\section{Acknowledgements}

I should like to thank Phil Diamond and Helen MacGillivray for helpful conversations, and Tony Pakes for drawing my attention to the work of Hsu and Wang. I should also like to thank both referees for valuable comments. This work was supported by the Australian Research Council. 


\section{References}

[1] S. E. Alm, "On the rate of convergence in diffusion approximations of jump Markov processes," Thesis, University of Uppsala, 1978.

[2] A. D. Barbour, "On a functional central limit theorem for Markov population processes", Adv. Appl. Probab. 6 (1974) 21-39.

[3] A. D. Barbour, "Quasi-stationary distributions in Markov population processes", Adv. Appl. Probab. 8 (1976) 296-314.

[4] A. D. Barbour, "Density dependent Markov population processes", preprint, Gonville and Caius College, Cambridge (1979).

[5] P. M. Diamond, "Stochastic models for interference between searching insect parasites", J. Austral. Math. Soc. Ser. B 30 (1989) 268-277.

[6] F. D. J. Dunstan and J. F. Reynolds, "Applications of stochastic processes to chemical reaction kinetics", Methods of Operations Research 33 (1978) 105-119.

[7] F. D. J. Dunstan and J. F. Reynolds, "Normal approximations for distributions arising in the stochastic approach to chemical reaction kinetics", J. Appl. Probab. 18 (1981) 263-267.

[8] D. T. Gillespie, "Exact stochastic simulation of coupled chemical reactions", J. Phys. Chem. 81 (1977) 2340-2361.

[9] P. Hall, "On the roles of the Bessel and Poisson distributions in chemical kinetics", $J$. Appl. Probab. 20 (1983) 585-599.

[10] J. Hsu and H. Wang, "Kinetics of bacterial adhesion - a stochastic analysis", J. Theor. Biol. 124 (1987) 405-413.

[11] D. W. Jordan and P. Smith, Nonlinear ordinary differential equations, (Oxford University Press, Oxford, 1977).

[12] T. G. Kurtz, "Solutions of ordinary differential equations as limits of pure jump Markov processes", J. Appl. Probab. 7 (1970) 49-58.

[13] T. G. Kurtz, "Limit theorems for sequences of jump Markov processes approximating ordinary differential processes", J. Appl. Probab. 8 (1971) 344-356.

[14] T. G. Kurtz, "Strong approximation theorems for density dependent Markov chains", Stochastic Process. Appl. 6 (1978) 223-240.

[15] R. M. May, Stability and Complexity in Model Ecosystems, 2nd ed., (Princeton University Press, 1974).

[16] D. A. McQuarrie, "Stochastic approach to chemical kinetics", J. Appl. Probab. 4 (1967) 413-478.

[17] B. G. Quinn and H. L. MacGillivray, "Normal approximations to discrete unimodal distributions", J. Appl. Probab. 23 (1986) 1013-1018.

[18] R. W. Parsons and P. K. Pollett, "Quasistationary distributions for some autocatalytic reactions", J. Statist. Phys. 46 (1987) 249-254.

[19] P. K. Pollett, "On the problem of evaluating quasistationary distributions for open reaction schemes", J. Statist. Phys. 46 (1989) 249-254.

[20] P. K. Pollett, "The generalized Kolmogorov criterion", Stochastic Process. Appl. 33 (1989) 29-44.

[21] N. G. van Kampen, Stochastic Processes in Physics and Chemistry, (North-Holland, Amsterdam, 1981).

[22] P. Whittle, "Statistical processes of aggregation and polymerization", Proc. Camb. Phil. Soc. 61 (1965) 475-495.

[23] P. Whittle, "Equilibrium distributions for an open migration process", J. Appl. Probab. 5 (1968) 567-571. 